\section{Building bridges: the emerging field of cardio-oncology}

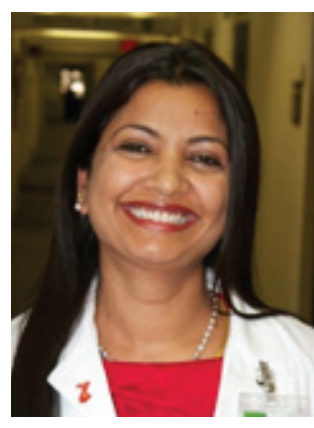

Susmita Parashar* speaks to Ellen Clarke, Commissioning Editor: Susmita Parashar is the Director, Winship at Emory Cardio-oncology Program at Emory University and is Associate Professor of Cardiology at Emory, and a cardiovascular outcomes researcher. Her research focuses on the role of clinical and biological factors in explaining outcomes of cardiovascular diseases in special high-risk population such as cancer and women. She has directed several clinical, translational, epidemiological and outcomes studies in cardiovascular patients. She has received several grants and awards including the Mentored Clinical Research Career Development Awards (K-12 and the K23) from the National Institute of Health and the Scientist Development Award (National) from the American Heart Association. Dr Parashar serves on the steering committee for the International Cardio-Oncology Society (ICOS), and is a member of the guidelines committee to develop a best practice consensus document for cardio-oncology at ICOS. She is also a steering committee member for the national cardio-oncology fellowship development as part of ICOS. She also serves on many professional organizations and committees at the institutional and national level including the Winship Cancer Institute Board. Dr Parashar has authored/coauthored over 60 peerreviewed publications, including invited textbook chapters, manuscripts, abstracts and review articles. Her work has been published in such prestigious journals as the New England Journal of Medicine, Archives of Internal Medicine and Circulation, and highlighted by the Nature and national media such as CNN, CBS and NPR news.

Q Thank you for taking the time to speak to us; could you tell us a little about your background \& work in the field of cardiology?

I am a clinical cardiologist at the Emory Heart and Vascular Center and associate professor of medicine (cardiology) at Emory University School of Medicine. Prior to joining faculty in the division of cardiology, I was an Assistant Professor of Medicine in the Division of General Internal Medicine at Emory for 8 years. I completed my training in internal medicine at Medical College of Georgia, Augusta and cardiology fellowship at Emory University. I completed a Master of Public Health and a Master of Science from Emory in 2005.

I am a cardiovascular outcomes researcher with emphasis on women and heart disease and cardio-oncology. My research focuses on the role of clinical and biological factors in

\section{KEYWORDS}

- cancer • cardio-oncology

- chemotherapy • heart disease

- radiation

*Emory University, 1365 Clifton Road NE, Building A, Atlanta, GA 30322, USA; smallik@emory.edu

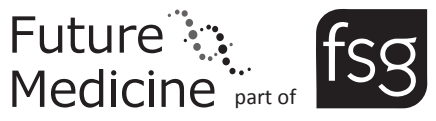


explaining outcomes of cardiovascular diseases in special high-risk population such as cancer. I closely collaborate with Winship Cancer Institute of Emory University toward our cardio-oncology program. I conduct research to describe the subclinical incidence and calculated risk for the prevalence of cardiomyopathy and coronary heart disease in cancer patients.

I serve on the steering committee for the International Cardio-Oncology Society (ICOS), and I am a member of the guidelines committee to develop a best practice consensus document for cardio-oncology at ICOS. I am also a steering committee member for the national cardio-oncology fellowship development as part of ICOS.

Q Can you tell us something about the origins of the cardio-oncology program at Emory University? Some information on the early background to this team of researchers?

During my training and practice in internal medicine and cardiology, I saw patients who had fought a great battle with cancer, and had survived due to the excellent care they received from their oncologists. Some of these patients developed heart disease as a late complication of their cancer treatment, including heart failure. Some of these patients were very young and had very young children. I found these stories heartbreaking. I wished there was some way we could provide care to these survivors sooner to prevent heart disease, minimize cardiovascular toxicity and improve their outcomes.

We developed an outstanding multidisciplinary team of clinicians and researchers at Emory to establish a unique cardio-oncology program to care for the heart health of cancer survivors. Our team includes Dr Natia Esiashvilli who is a radiation oncologist and directs the Young Adult Cancer Survivor Program with her outstanding nurse practitioner Mary Batcha. Dr Christopher Flowers is a clinical oncologist and director of Winship Lymphoma Program. Joan Giblin is the director of Adult Survivorship Program and has been our advisor and strong collaborator from the very beginning. Our team also includes Dr Carolyn Reilly from the School of Nursing at Emory. The proactive approach of the multidisciplinary Winship at Emory Cardio-Oncology Program is to provide care to patients in varying stages of cancer treatment who have or are at risk for developing heart disease; and to conduct high-quality research to improve heart health of cancer patients.
Q The program obviously involves a strong level of collaboration between cardiologists \& oncologists; are there any challenges that are particular to putting together a successful team in this field?

We have a very dedicated cardio-oncology team at Emory. The main challenge in putting together a successful team in this very novel field was to find passionate collaborators who have time and resources to be actively engaged in this novel program. I was fortunate to have a strong support of my cardiology chair, Dr Robert Taylor, and protected time to establish this program.

I sought out and met several oncologists at Emory to seek collaboration. All of them uniformly wanted the best care and outcomes for our patients. I felt that at times, due to a bigger concern regarding immediate cancer treatment, some oncologists may have been concerned that proactively highlighting cardiotoxic potential of chemotherapy and radiation therapy, defining cancer patients who are receiving chemotherapy as a high-risk cardiovascular patient, and referring patients upfront to a cardiologist may increase patients' refusal rates for cancer treatment, lead to patients' preferring a less effective therapy for cancer, de-emphasize their focus on cancer treatment, add to their perceived disease and financial burden and lead to unnecessary adverse psychosocial outcomes. Lack of nationally standardized clinical guidelines and clinical management tools made the collaboration further challenging.

As I mentioned above, fortunately, we found a great team consisting of Giblin and Drs Esiashvilli, Flowers and Reilly. We began by conducting small clinical research projects in this area approximately 4 years ago and further solidified our collaboration to develop a clinical and research cardio-oncology program. We decided to base the clinical cardio-oncology program in preventive cardiology to alleviate any concerns patients and their oncologists may have in seeking cardiovascular care since they may be concerned about labeling these patients as 'heart disease patients.'

Giblin was very instrumental in identifying high-risk survivors in her adult survivorship clinic and began referring these patients to the cardiooncology clinic. Some of these patients were such high risk that they ended up getting bypass surgery or stents within a week of seeing me. Others were delighted to get excellent personally tailored preventive care in our program. Based on their very enthusiastic input and satisfaction, we 
developed collaboration with a very unique Young Adult Survivor Program at Emory and lymphoma clinics as well as inpatient oncology services. We are also seeing pregnant women who have survived cancer and have received chemotherapy and radiation therapy.

Having a uniform echocardiography protocol is very important to our program. We have developed and piloted an echocardiography protocol for oncology patients. Our next undertaking and challenge is to provide training and implement the protocol at all our sites.

We presented our results from the clinical program and research at institutional and national meetings. I also gave lectures on this topic and our cardio-oncology program at Emory and other national meetings.

Subsequently, we presented our program to Dr Robert Taylor, chair of cardiology and Dr Walter Curran, executive director of Winship, who welcomed the formal establishment of this program at Emory.

\section{Q The cardio-oncology program at Emory is} one of the few programs in the USA dedicated to addressing the cardiovascular side effects of cancer therapy. Why do you think it is that this important area is somewhat under-represented in terms of specific research programs?

Cardiologists and oncologists who work in this novel field face a number of challenges. One of these challenges is the fact that there are relatively few standardized clinical guidelines, clinical management tools or training programs devoted to the field of cardio-oncology. Consequently, clinicians and researchers in this field operate from their personal or institutional experiences or guidelines rather than proven standardized guidelines.

Through the ICOS, we have developed a best practice consensus document for cardio-oncology. This document is in the process of being published. Cardiologists and oncologists need to communicate and collaborate effectively to provide optimal care. ICOS and the American College of Cardiology are doing tremendous work in collaboration of these two very diverse specialties.

\section{Q What are the short-term goals of the} research conducted at Emory? What ongoing work do you feel has the greatest possibility of benefitting patients in the coming years?

We are conducting research for early detection and monitoring of cardiotoxicity. Recent studies have demonstrated that asymptomatic decreased left ventricular ejection fraction (LVEF) can lead to a markedly increased risk of the development of heart failure and death, and is a class I indication for therapy with $\beta$-blockers and ACE inhibitors according to American College of Cardiology/American Heart Association. Some recent studies by Cardinale and others indicate that in anthracycline-related cardiomyopathy, LVEF recovery and cardiac event reduction may be achieved when asymptomatic cardiac dysfunction is detected early and modern heart failure treatment, including $\beta$-blockers and ACE inhibitor, is promptly initiated. The likelihood of LVEF recovery is greater for patients who had treatment initiated within 2 months from the end of anthracycline-based chemotherapy. After 2 months, this proportion progressively decreased, and incomplete LVEF recovery was observed among those who had therapy initiated only after 6 months. This emphasizes the crucial importance of early detection and management of cardiotoxicity in cancer patients.

Our current research focuses on the shortterm goal of finding strategies and clinical management tools to improve surveillance of cancer survivors and to identify early subclinical markers of cardiotoxicity in patients who have or are receiving anthracycline-based chemotherapy and/or radiation therapy. Specifically, we are evaluating the prognostic significance of early echocardiographic markers of cardiotoxicity such as strain-based parameters on cardiovascular outcomes of cancer patients. If we can identify early markers of cardiotoxicity, we can perhaps target these patients more aggressively in terms of cardiovascular surveillance and management, in order to improve their outcomes.

\section{Q Conversely, what long-term projects} do you feel are being carried out at Emory that may not have an immediate effect on therapeutic practice but present very novel approaches in managing cardiovascular health in cancer patients?

The number of cancer survivors in the USA is currently estimated at over 13 million and is expected to increase significantly due to earlier diagnosis, and advances in treatments that include anthracycline-based chemotherapy and radiation therapy. However, these agents also lead to significant cardiotoxicity and the impact of anthracyline-based chemotherapy and 
radiation therapy on long-term health of these survivors is substantial.

Our long-term project is to examine early subclinical markers of long-term consequences of anthracyclines and radiation therapy and to streamline and coordinate a multidisciplinary cardio-oncology program developed based on patients' concerns, values, preferences and challenges. Our long-term goal is to develop a uniform way of providing care to patients in varying stages of cancer treatment who have or are at risk for heart disease. Our goal is to create a feasible and sustainable multidisciplinary management team that includes cardiologists and oncologists, nurse practitioners, nurses, social workers and echocardiographers.

I am also very delighted about a recent collaboration. We have begun collaborating with the Children's Healthcare of Atlanta Aflac Cancer Center and the Sibley Heart Center, towards cardio-oncology research in young cancer survivors. Our goal is to streamline a seamless transition from the pediatrics survivorship to adult survivorship program at Emory. Through this collaboration, we are also planning to participate in some multi-center cardio-oncology trials.

Q How did you personally come to be interested in cardio-oncology from a cardiology background?

Prior to my cardiovascular training, I was an assistant professor in general medicine at Emory. During that time and my training in cardiology, I saw many patients who had survived cancer and now, sometimes after years, had complications of chemotherapy or radiation therapy, including heart failure, coronary artery disease or arrhythmias. Many of these patients had not followed up with any doctor for years. Many of them were very young having received chemotherapy when they were children. I found such stories very concerning. I wish there was something we could do for these survivors. In addition, I routinely read echocardiographs of patients going through chemotherapy. I came across several cases where the patients' ejection fraction was now low after chemotherapy, due to which they were no longer candidates for some life-saving treatments. Again, I wondered if the left ventricular dysfunction could be prevented. One of my key interest and projects had been on women and heart disease. I sought out collaboration with breast oncologists to combine both of my interests and gradually developed programs to include both men and women, and all kinds of cancer.

Q In your opinion, how underestimated/ underappreciated are cardiovascular side effects of cancer treatment in terms of both physician \& public awareness?

Cardiotoxicity caused by certain chemotherapy and radiation therapy is an underestimated problem in my opinion, in terms of both physician and public awareness. As cardiologists, we do not see these patients till they have some adverse cardiac consequences. Since there are no concrete guidelines on the follow-up of these patients, oncologists may be more focused on treating their cancer, rather than late effects. Especially patients without history of cardiac disease are often treated as outpatients and therefore without cardiac monitoring. Consequently asymptomatic and symptomatic cardiac problems may be overlooked. The benefit of prophylactic agents remains unclear, so close cardiac monitoring is the most established method to prevent manifest cardiotoxic events.

\section{Q Could you give us something of an} overview of your own current research projects? What stages are they in \& how are results progressing?

We are conducting clinical research in this field for past 4 years. We conducted a retrospective cohort study of patients with aggressive B-cell non-Hodgkin lymphoma who were treated with anthracyclines to assess the cardiac surveillance and heart failure management of these patients at Emory prior to the development of a comprehensive cardio-oncology program. We found that only a third of patients who received anthracyclines had imaging surveillance prior to and after receiving chemotherapy; out of these, a third developed cardiomyopathy. We also found that a minority of patients who developed cardiomyopahty were referred to a cardiologist, started on heart failure medications such as $\beta$-blockers or ACEI or had repeat imaging for further LVEF evaluation. These results were presented at the American Heart Association meeting last year and the manuscript is being considered for publication.

Our second study prospectively evaluated subclinical markers of cardiac dysfunction including novel echo parameters, coronary atherosclerosis and inflammation in patients with 
lymphoma 2-10 years after receipt of anthracycline chemotherapy and radiation. We are analyzing these data and will be presenting the results soon. We are also applying for grants to conduct other cardio-oncology research.

\section{Q What do you feel is the most pressing} issue for patients \& clinicians in the field of cardio-oncology?

I feel that the most pressing issue for patients and clinicians in the field of cardio-oncology is the urgent need for development of standardized clinical guidelines, clinical management tools or training programs devoted to the field of cardio-oncology. We need standardized guidelines regarding surveillance, monitoring and treatment of patients in varying stages of cancer treatment including chemotherapy, radiationtherapy and neoadjuvant therapy. Through the ICOS, we have developed a best practice consensus document for cardio-oncology. This document is in the process of being published.

\section{Q What studies in recent years do you feel have helped to advance the field the greatest?}

Several recent studies have set critical groundwork in the field including cardiologists at oncology-dedicated institutions such as MD Anderson and Memorial Sloan Kettering Center in the USA or Istituto Europeo di Oncologia in Milan, Italy to name a few. Recent studies by Dr Cardinale have demonstrated a crucial need to detect cardiotoxicity early. Her team showed that in patients with anthracycline-related cardiomyopathy, LVEF recovery and cardiac event reduction may be achieved when asymptomatic cardiac dysfunction is detected early and heart failure treatment, including $\beta$-blockers and ACE inhibitor, is initiated. The sooner the initiation of these heart failure therapies, the better the outcome. Thavendiranathan et al. recently demonstrated the value of echocardiographic myocardial deformation parameters for the early detection of myocardial changes and prediction of cardiotoxicity in patients receiving cancer therapy. I believe that early detection of cardiotoxicity is of paramount importance so that early therapy may be instituted.

Recently, American Society of Echocardiography and European Association of Cardiovascular Imaging have published an expert consensus document of multimodality imaging of adult patients during and after cancer treatment that is a very useful clinically relevant document.

Q In your view, what do the next few years hold for the field? Do you see programs similar to Emory's becoming more common/ an increased focus on cardio-oncology at research conferences?

The Winship Cardio-Oncology Program, a joint collaboration of Emory University Division of Cardiology and Winship Cancer Institute, is one of the few in the USA dedicated to address the cardiovascular side effects of cancer therapy and improving cardiovascular outcomes for cancer survivors. Over the next few years, more centers will launch such programs to provide care to patients in varying stages of cancer treatment who have or are at risk for heart disease. The specialty cardio-oncology clinics will provide collaborative evaluation and care of cancer patients receiving chemotherapy, radiation therapy and neoadjuvant therapy. The objectives of these clinics will be to provide expert advice and guidance for patients who are either undergoing cancer treatment, or have completed cancer treatment to aid in the prevention, monitoring and management of cardiovascular toxicities with the goal that a cancer survivor of today does not become the cardiovascular disease patient of tomorrow.

We have a cardio-oncology working group through the ICOS. Cardio-oncologists from USA and internationally meet virtually once a month to discuss cases as well as protocols. For example, at Emory, we recently had a challenging case of a cancer patient with very advanced heart disease including heart failure with ejection fraction $30 \%$ and severe multivessel coronary disease. We were consulted to provide input on the choice of chemotherapy. I posted this very challenging question to our ICOS group which has $>150$ members. I received $>30$ replies from cardiologists and oncologists worldwide which tremendously helped care of this patient. I foresee more working collaborative groups dedicated to this cause. These collaborations will lead to further multidisciplinary research at national and international levels, and development of standardized guidelines. Recently, the American College of Cardiology and the American Society of Clinical Oncology have begun to collaborate on a number of projects, and this year's American College of Cardiology meeting included several working groups devoted to cardio-oncology. 
Q Similarly, over the longer term, what do you think might be the most dramatic changes that will occur in managing cardiovascular health in cancer patients? Over the next several years, cardio-oncology will likely be identified as a subspecialty with dedicated training in terms of cardio-oncology fellowships. There will be guidelines and consensus documents that will help clinicians provide the best care to cancer patients to improve their cardiovascular outcomes. There will be more and more research in this area, more funding and more recognition of the importance for this specialty area.

\section{Disclaimer}

The opinions expressed in this interview are those of the interviewee and do not necessarily reflect the views of Future Medicine Ltd.

Financial \& competing interests disclosure

The author has no relevant affiliations or financial involvement with any organization or entity with a financial interest in or financial conflict with the subject matter or materials discussed in the manuscript. This includes employment, consultancies, honoraria, stock ownership or options, expert testimony, grants or patents received or pending, or royalties.

No writing assistance was utilized in the production of this manuscript. 